\title{
Analyzing Effect of Profile Change of Top Surface of 60kg Rail upon Vehicle Dynamics
}

\author{
Atsushi SHIMIZU \\ Track Geometry \& Maintenance Laboratory, Track Technology Division \\ Tadanobu IIDA \\ Vehicle Dynamics Laboratory, Vehicle Structure Technology Division
}

\begin{abstract}
To evaluate running stability, equivalent conicity was computed by considering the combination of a new rail profile and arc wheel profile (for Shinkansen). The new rail profile was obtained by applying the radius of curvature of the top surface of a JIS $50 \mathrm{kgN}$ rail, $300 \mathrm{~mm}$. The computation results when using the new rail showed small equivalent conicity and high running stability regardless of the degree of wear of the wheel. Furthermore, results from vehicle dynamics simulations showed that the new rail was endowed with running characteristics equivalent to those of JIS 60kg rail, and no evidence was found of any significant influence on running safety.
\end{abstract}

Keywords: JIS 60kg rail, equivalent conicity, hunting, vehicle dynamics, arc wheel profile

\section{Introduction}

When the Tokaido Shinkansen was initially opened in 1964, a conical wheel profile was used as the wheel profile (for Shinkansen), whereas for more recent vehicles, the arc wheel profile is employed. Tracks, on the other hand were originally laid with $50 \mathrm{kgT}$ rail [1] whose rail profile was designed in consideration of the initial conical wheel profile, followed by JIS $60 \mathrm{~kg}$ rail [2] until most recently which has the same rail head profile as the $50 \mathrm{kgT}$ rail. Consequently, vehicles today are running on tracks with attributes dating back to the time when the rails were first designed.

Therefore, in order to improve the running safety and running stability associated with the planned increases in speed of the future, it may be necessary to consider a new rail profile in accordance with the arc wheel profile. Accordingly, following measurements of the wheel and rail profiles from in-service lines, with particular focus on equivalent conicity as an evaluation index of running stability, this study investigated new rail profiles with higher running stability. This paper describes that the result of these investigations.

\section{Equivalent conicity and hunting}

\subsection{Equivalent conicity}

The wheels of the railway vehicle, different from freely moving wheels on a flat road surface, such as an automobile, are guided by two rails, and the right and left wheels are rigidly coupled. Railway wheels are designed with a gradient toward the outer edge, moving away from the flange part, which forces the left and right wheels to re-center along a straight line. In curves, this difference between effective radii of the left and right wheels, allows smooth negotiation of the curve.

The left and right wheel contact angles and radii of rotation differ in both the rail and wheel profiles; the rate at which the radii of rotation on the left and right wheels changes, is equivalent to the tread gradient. For example, in the case of a wheelset modelled as a perfect cylinder, with a zero gradient, the difference in rotational radii would be zero, and the tread gradient would also became a constant zero. In the case where rail and wheel profiles combine forming an arc of multiple curvatures, the tread gradient varies with the rail/wheel contact position. Equivalent conicity $\gamma_{e}$ used for the evaluation of running stability in this paper, was approximated by a linear constant, the geometric contact gradient caused by the curvature of the rail head and tread gradient of the wheel.

Equivalent conicity $\gamma_{e}$ is determined by the following procedure.

A) From the profile data of the rails and the wheels, calculate wheel radii of the left and right at the contact position with the rail when the wheelset is moved from/to the left and right.

B) Using the data of (A) and the simultaneous differential equations (Eq. (1), (2)) determine responses of rolling on rails sequentially calculated by giving the initial displacement $(3 \mathrm{~mm})$ in the lateral direction. Figure 1 shows the symbols used for the calculation.

$$
\begin{aligned}
& d y_{w} / d t=V \cdot \varphi_{w} \\
& d \varphi_{w} / d t=\frac{\left(r_{L}-r_{R}\right)}{r_{0}} \cdot \frac{V}{2 b}
\end{aligned}
$$

C) Obtains $S_{1}$ (hunting wavelength of one wheelset) from the left and right responses of wheelset, to determine the equivalent conicity $\gamma_{e}$ by substituting (3).

$$
\gamma_{e}=4 \pi^{2} \cdot \frac{b r_{0}}{S_{1}^{2}}
$$




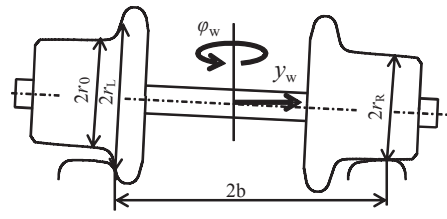

where $y_{w}=$ left and right displacement of the wheelset;

$\varphi_{w}$ : yaw angle of wheelset;

$V:$ speed;

$\mathrm{r}_{\mathrm{L}}, \mathrm{r}_{\mathrm{R}}$ : radii at the rail contact position of the left and right wheels;

$\mathrm{r}_{0}$ : radius at center valve position; and

$\mathrm{b}:$ a half of wheel contact point interval.

Fig. 1 Load-deflection relationship of slabs

\subsection{Hunting}

A phenomenon called "hunting" sometimes occurs on railway vehicles causing instability. This is when the bogie and wheelset begin to sway violently from side to side at high speed. The basic cause for hunting is related to the wheel tread having a gradient. The difference in left and right wheel radii generates the tread gradient leading the wheels to meander from side to side with a constant amplitude. That is $S_{1}$ obtained in 2.1. When equivalent conicity is small, the wheel contact point interval and wheel radius are large, $S_{1}$ becomes longer.

Increasing the length of the $S_{1}$ oscillation is one of the approaches to suppress hunting. Reducing the equivalent conicity, and increasing the radius of the wheel can therefore be considered as a strategy to reduce hunting. When

Table 1 Stability of hunting and curving performance

\begin{tabular}{|c|c|c|}
\hline Viewpoint & Stability of hunting & Curving performance \\
\hline Bogie rotational resistance & Large & Small \\
\hline $\begin{array}{c}\text { Axle box suspension } \\
\text { stiffness }\end{array}$ & Partly large & Small \\
\hline Equivalent conicity & Small & Large \\
\hline
\end{tabular}

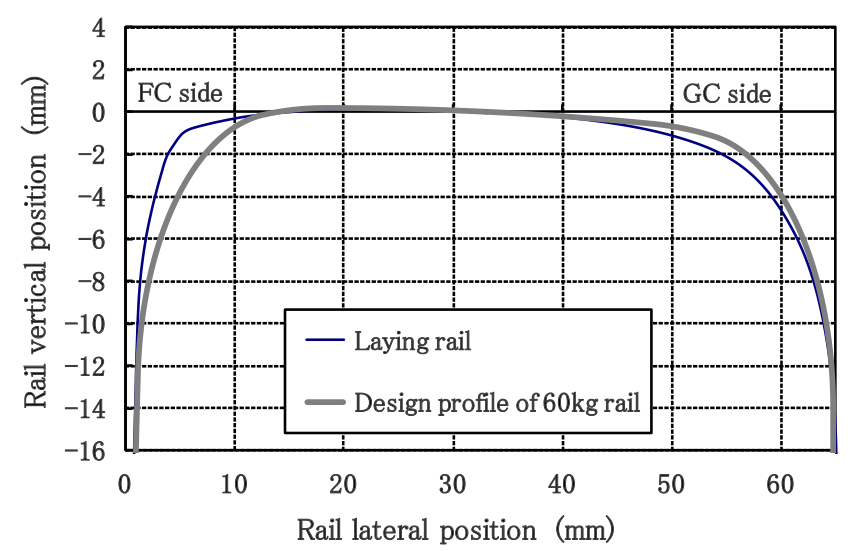

(a) Rail profile considering the arc wheel profile, the gradient increases as the gauge narrows, which means that if the gauge is widened, the wheel/rail contact point can be kept in the lower gradient area thereby helping to suppress hunting. Further stability can be achieved against hunting by increasing the natural frequency, either by reducing the mass of the wheelset, or by raising its support rigidity.

However, in order to pivot the bogie smoothly through a curve and reduce lateral forces, running performance through curves must be improved, namely by reducing the bogie rotational resistance or increasing equivalent conicity. Thus, requirements for stabilizing hunting and for curve running performance are conflicting. Table $1 \mathrm{ex}-$ plains and summarizes them.

\section{Measurement of rail and wheel profile}

\subsection{Rail profile}

An instrument (Miniprof) was used to measure the rail head shape of an in-service Shinkansen line. Measurements were taken on left and right rails in a straight section, the radius of curvature and rail profile were calculated for sections without weldings.

Figure 2 shows the rail profile and radius of curvature of the rail head at the place where cumulative passing tonnage was 530 million tons. It also shows the design profile of the JIS $60 \mathrm{~kg}$ rail, and the design radii of curvature of JIS $60 \mathrm{~kg}$ rail and JIS $50 \mathrm{kgN}$ rail. Although the design radii of curvature of the JIS $60 \mathrm{~kg}$ rail head is $600 \mathrm{~mm}$, the radius of curvature of the laid rail head is smaller than the design value, about $300 \mathrm{~mm}$ on average, which is close to the design value of $50 \mathrm{kgN}$ rail.

\subsection{Wheel profile}

In order to understand the wear status of the wheel tread on Shinkansen vehicles, the wheel profiles of two vehicles with different wheel design profiles were measured at various mileages. The wheel tread shape for each mileage was calculated using the wheel wear shape estimation tool [3] based on this data.

Figure 3 shows the wheel tread state when it has reach

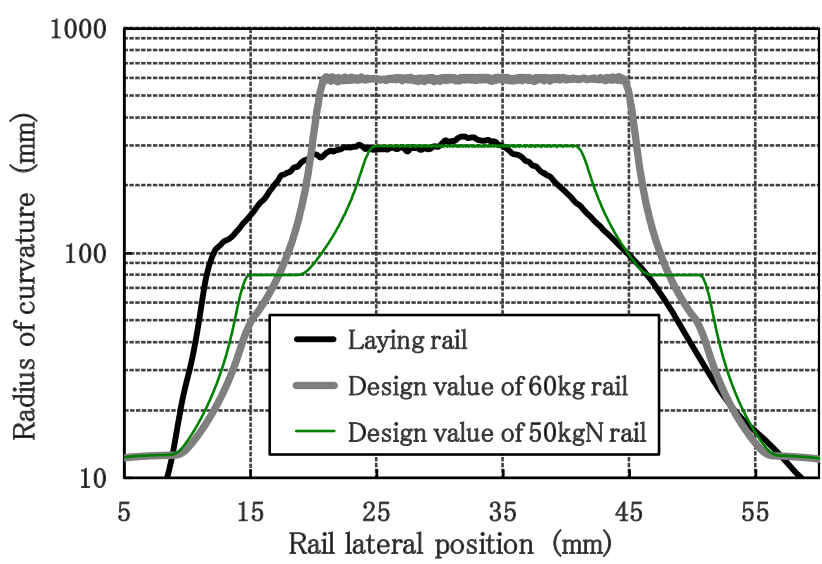

(b) Radius of curvature of rail head

Fig. 2 The rail profile and radius of curvature of rail head 


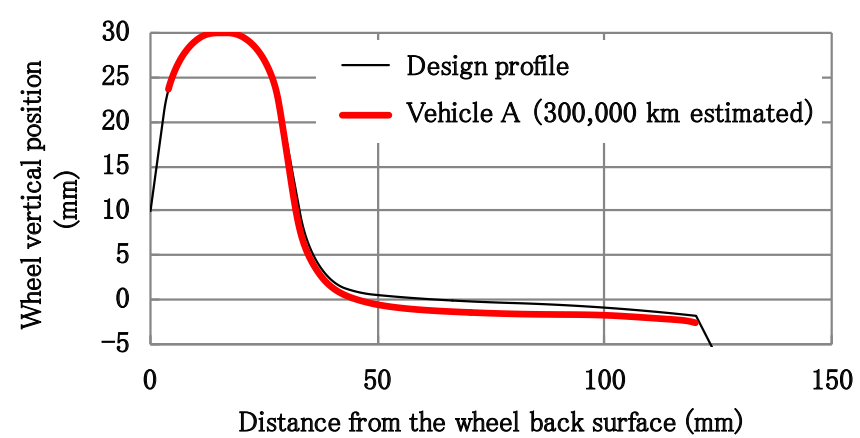

Fig. 3 Wheel tread shape of vehicle A

its reprofiling period compared with the design profile. This figure illustrates that tread wear does not necessarily proceed uniformly and tread wear is concentrated on the tread surface at a distance of $60 \sim 80 \mathrm{~mm}$ from the wheel back. Figure 4 shows tread wear progress on the wheel of vehicle A. Note that there is less wear on vehicle B than on vehicle A. Furthermore, Fig. 5 shows the equivalent conicity calculated by combining the design profile of JIS $60 \mathrm{~kg}$ rail and estimated wheel wear. In this case, equivalent conicity was calculated by obtaining the $\mathrm{S} 1$ from the simulation of a single wheelset.

As vehicle A mileage increases, so does equivalent conicity. In the case of vehicle $B$, equivalent conicity increased by about 0.1 . Figure 6 shows the contact point range for vehicle A between the JIS $60 \mathrm{~kg}$ rail and the wheel (design profile and $300,000 \mathrm{~km}$ estimated profile) when the wheelset is displaced $\pm 3 \mathrm{~mm}$. As the wheel tread wears, contact between the rail and that tread becomes difficult, and in comparison with design profile of wheels the contact area grows. Since the turning radii difference between the left and right wheels is larger when contact area of the wheel expands, equivalent conicity increased in vehicle $A$ as mileage rose. This trend became more apparent as the radius of curvature of the rail head surface increased. In the case of vehicle B, since there was less wheel tread wear than for vehicle A, equivalent conicity did not increase. Although the causes underlying the difference in amount of wear between vehicle types was not examined in this study, it is posited that differences in running along the line sections and tread polishing could be factors.

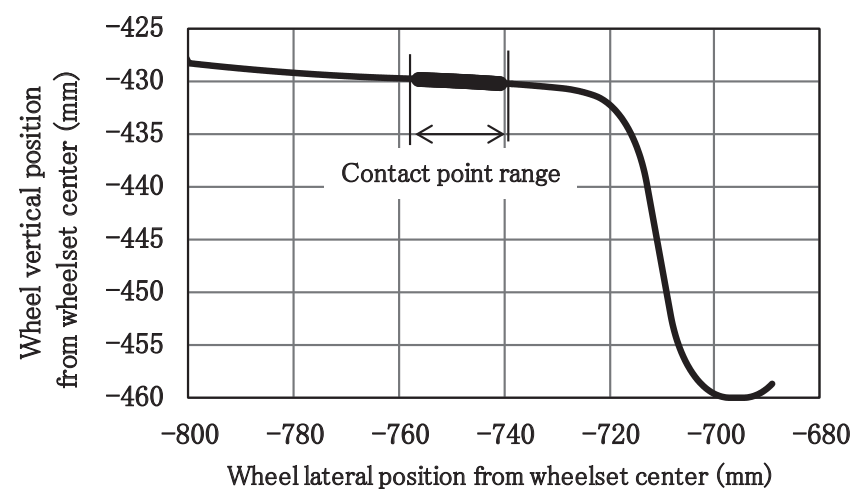

(a) Design wheel profile

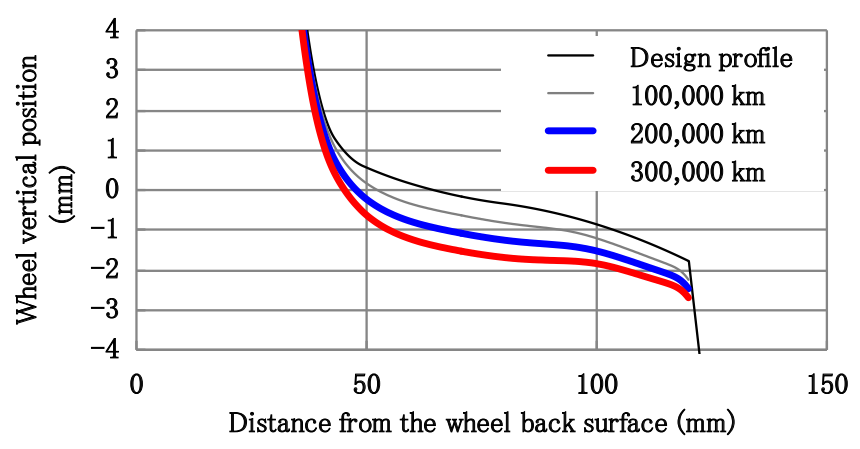

Fig. 4 Tread wear progress of wheel on vehicle A

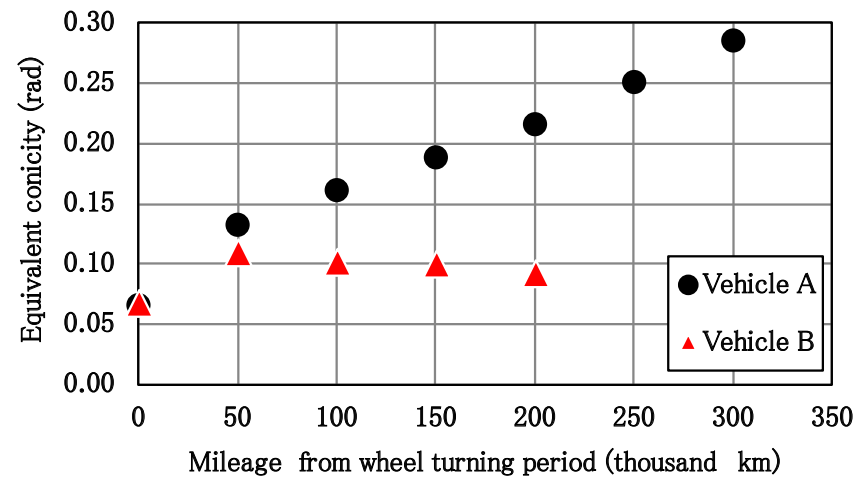

Fig. 5 Equivalent conicity calculated by combining design profile of JIS $60 \mathrm{~kg}$ rail and estimated wheel wear for vehicle $A$

\section{Consideration of the new rail profile}

\subsection{Examination of running stability and design of the new rail profile}

Upon the design of the new rail profile, a rail profile creation program was developed which can accept any radius of curvature and create a candidate profile. As indicated in Section 3.1, considering that the radius of curvature $R$ of the rail head surface is smaller than the design value of JIS $60 \mathrm{~kg}$ rail, a candidate profile was created by gradually reducing the design value of JIS $60 \mathrm{~kg}$ rail $(\mathrm{R}=$ $600 \mathrm{~mm}$ ). Then, for each candidate profile, the equivalent



(b) $300,000 \mathrm{~km}$ estimated wheel profile

Fig. 6 Contact point range with JIS $60 \mathrm{~kg}$ rail when the wheelset is displaced $\pm 3 \mathrm{~mm}$ 


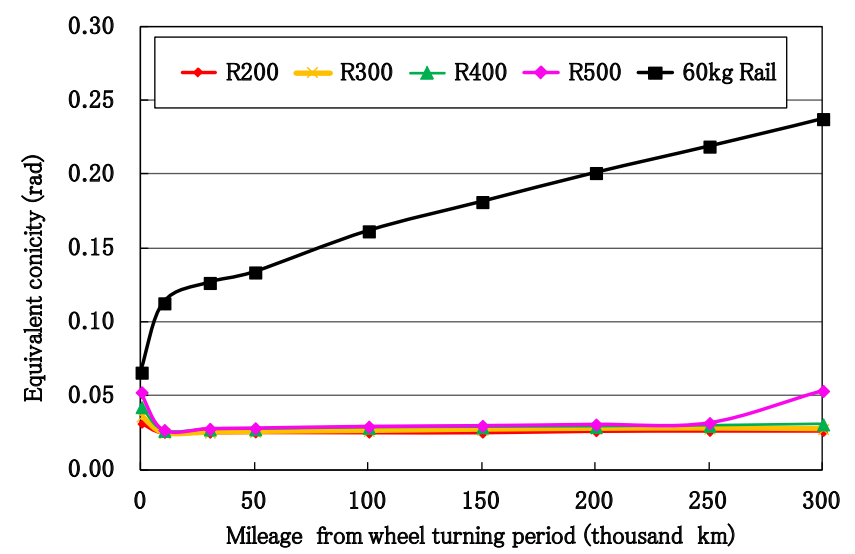

(a) Vehicle A

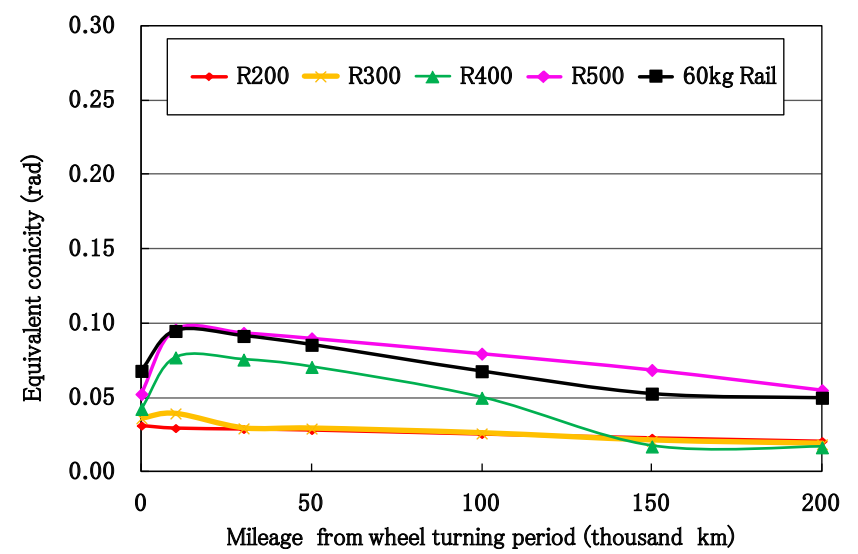

(b) Vehicle B

Fig. 7 Equivalent conicity combining candidate rail profiles and wheels from both vehicle types

conicity was calculated by combining worn wheel profiles estimated from the results of 3.2. For the proposal of the new profile, a relative evaluation was made starting from the combination of a JIS 60kg rail and arc wheel profile (for Shinkansen) as a base, while varying candidate profiles.

Figure 7 shows the equivalent conicity for combinations of candidate rail profiles and vehicle type wheels. While preserving the shape of the GC (gauge corner) side upper corner of the head $(\mathrm{R}=13 \mathrm{~mm})$ unchanged, candidate profile shapes were created by changing the curvature radius and the width of the center of the top surface, and the curvature radius of the transition zone up to $R=13 \mathrm{~mm}$.

Results for vehicle A showed that equivalent conicity increased with mileage only in the case of JIS $60 \mathrm{~kg}$ rail. Results for vehicle B showed that equivalent conicity was higher for profiles with $\mathrm{R}=400 \mathrm{~mm}$ or more. However, contrary to the combination of JIS $60 \mathrm{~kg}$ rail and vehicle A, there was no tendency for equivalent conicity to increase with mileage. For both vehicles results indicated that equivalent conicity was substantially the same for profiles with a radius of curvature of $300 \mathrm{~mm}$ and $200 \mathrm{~mm}$, and variation of the equivalent conicity along with mileage was small. Therefore equivalent conicity was calculated for some of the candidate profiles with a radius of curvature under $300 \mathrm{~mm}$. This revealed that the increase in equivalent conicity was small, and the same was found for candi- date profiles with a radius of curvature of $400 \mathrm{~mm}$ and 500 $\mathrm{mm}$. From consideration of the above results, in order to improve running stability by reducing equivalent conicity, it is best for the rail head surface radius of curvature to be below $300 \mathrm{~mm}$. These results therefore point to a recommended rail profile with a rail head curvature of radius of under $300 \mathrm{~mm}$, for several reasons: firstly in the case of a radius of below $300 \mathrm{~mm}$, the change in equivalent conicity due to wheel wear is very low, secondly, this is close to the curvature of radius for JIS $60 \mathrm{~kg}$ railhead in a state of wear, and thirdly, since it is almost equivalent to the curvature of radius of the traditional JIS $50 \mathrm{kgN}$ rail, it can be considered for application to conventional lines as well.

\subsection{Study of running safety}

In order to examine the running safety when using the new rail profile, vehicle dynamic simulations were performed [4]. Vehicle A with a motor was used as the model. Derailment coefficients were calculated for running in a straight section with track irregularities (alignment) and a curved section without irregularities. Three sine waves were used and amplitude and wavelength were changed to simulate irregularities.

Figure 8 and 9 shows the relationship of the maximum value of derailment coefficient and track irregularity for a

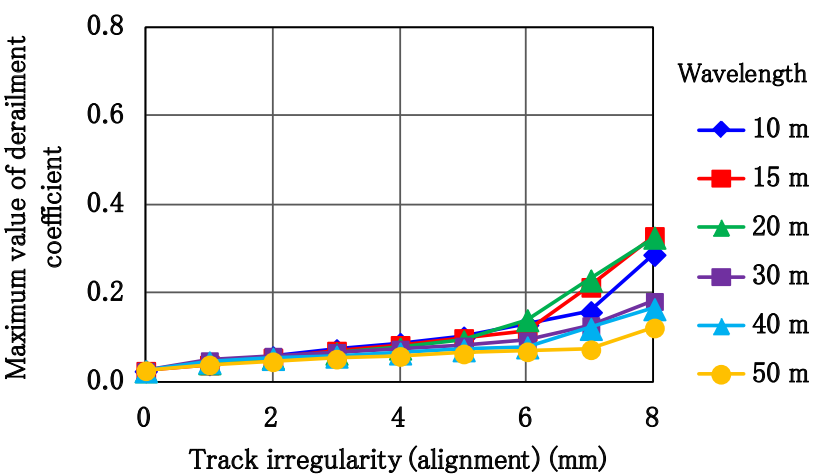

(a) JIS 60kg rail



(b) New profile rail

Fig. 8 Relationship between maximum value of derailment coefficient and track irregularity (Design wheel profile) 


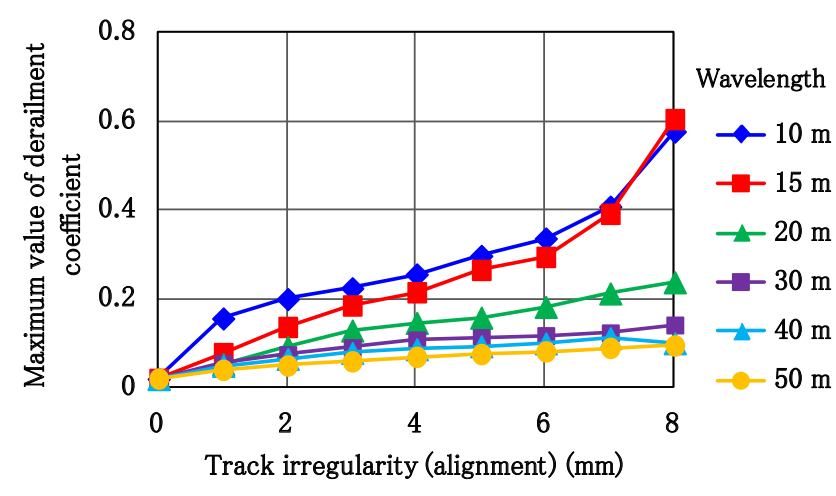

(a) JIS $60 \mathrm{~kg}$ rail

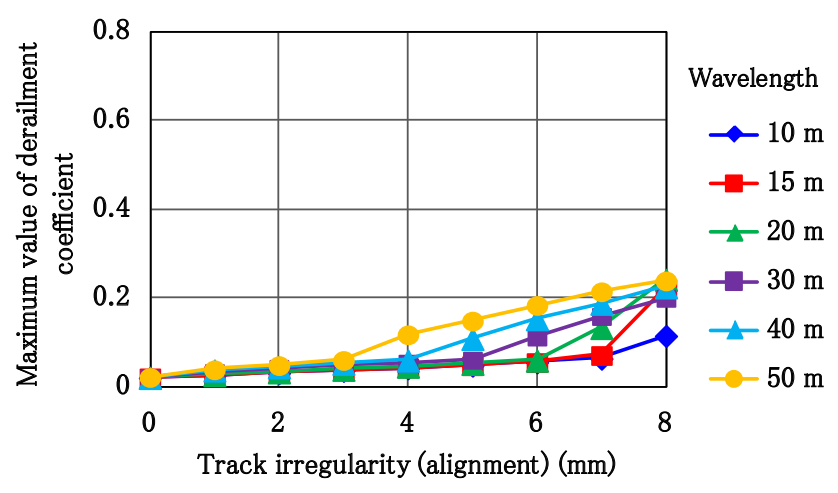

(b) New profile rail

Fig. 9 Relationship between maximum value of derailment coefficient and track irregularity (300,000 km estimated wheel profile)

speed of $320 \mathrm{~km} / \mathrm{h}$ on a straight section. In case of JIS $60 \mathrm{~kg}$ rail, when the wavelengths were $10 \mathrm{~m}$ and $15 \mathrm{~m}$, the derailment coefficient was greater than for other wavelengths when the amplitude rose above $6 \mathrm{~mm}$. This trend became more apparent in the case of worn wheels. On the other hand, in the case of the new rail profile, when the wavelengths were $10 \mathrm{~m}$ and $15 \mathrm{~m}$, the derailment coefficient was smaller than the JIS $60 \mathrm{~kg}$ rail profile, whereas the derailment coefficient was slightly larger than JIS $60 \mathrm{~kg}$ rail in the case of $40 \mathrm{~m}$ and $50 \mathrm{~m}$. However, since the derailment coefficient in any of these conditions reached only 0.24 at most, this poses no practical problem in terms of running safety. Table 2 shows the outside derailment coefficients for a curve of radius $4000 \mathrm{~m}$ with no track irregularities and a cant of $155 \mathrm{~mm}$. From this table, since no significant difference can be observed in the derailment coefficient, it can be considered that the difference in curve negotiation performance between the new rail profile and JIS $60 \mathrm{~kg}$ rail is very small for high-speed sections of Shinkansen line.

Table 2 Outside derailment coefficients for a curve of radius $4000 \mathrm{~m}$

\begin{tabular}{|c|c|c|c|}
\hline \multirow{2}{*}{ Wheel } & \multirow{2}{*}{ Rail } & \multicolumn{2}{|c|}{ Speed } \\
\cline { 3 - 4 } & & $275 \mathrm{~km} / \mathrm{h}$ & $320 \mathrm{~km} / \mathrm{h}$ \\
\hline \multirow{2}{*}{ Design profile } & JIS 60kg rail & 0.10 & 0.14 \\
\cline { 2 - 4 } & New profile rail & 0.09 & 0.16 \\
\hline $\begin{array}{c}300,000 \mathrm{~km} \\
\text { estimated profile }\end{array}$ & JIS 60kg rail & 0.07 & 0.15 \\
\cline { 2 - 4 } & New profile rail & 0.09 & 0.16 \\
\hline
\end{tabular}

\section{Authors}



Atsushi SHIMIZU

Research Engineer, Track Geometry \& Maintenance Laboratory, Track Technology Division

Research Areas: Carbody Vibration Control, Ride Comfort Evaluation

\section{Conclusions}

To evaluate Shinkansen running stability at high speed equivalent conicity was computed considering a combination of the new rail profile and arc wheel profile (for Shinkansen).

The results of this study show that the new rail profile should have a curvature of radius of the rail head of 300 $\mathrm{mm}$, equivalent to the JIS $50 \mathrm{kgN}$ rail. Computation results from trials using the new rail show small equivalent conicity and high running stability regardless of the degree of wear of the wheel.

Furthermore, vehicle dynamics simulations revealed that the new rail is endowed with running characteristics equivalent to JIS $60 \mathrm{~kg}$ rail, and no evidence was found of any influence on running safety.

\section{References}

[1] Sasaki, N., "Design of the new profile rail," Railway Technical Research Report, No. 20, 1961(in Japanese).

[2] Watanabe, K. and Sugiyama, T., "The Design of JIS 60kg Rail and Fish-Plate," Quarterly Report of RTRI, Vol. 10, No. 2, pp. 71-72, 1969.

[3] Haga, A., "Trend of wheel wear and wheel lifetime estimation," Transactions of the Japan Society of Mechanical Engineers, Vol. 113, No. 1094, pp. 32-34, 2010(in Japanese).

[4] Sato, A., "Study on Dynamics of an Independent Wheel Bogie with Steering Device for Railway Vehicle," RTRI Report, No. 37, 2000 (in Japanese).

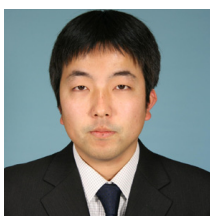

Tadanobu IIDA

Research Engineer, Vehicle Dynamics Laboratory, Vehicle Structure Technology Division Research Areas: Vehicle Dynamics 